\title{
Endovascular Recanalization of an Infrarenal Aortoiliac Occlusion Using a Combined Bilateral Subintimal Approach
}

\author{
Nikolaos Galanakis ${ }^{1}$, Nikolaos Kontopodis ${ }^{2}$, Elias Kehagias ${ }^{1}$, Nikolaos Daskalakis $^{2}$, \\ Christos V. loannou ${ }^{2}$, and Dimitrios Tsetis ${ }^{1}$ \\ ${ }^{1}$ Interventional Radiology Unit, Department of Medical Imaging, ${ }^{2}$ Vascular Surgery Unit, Department of Cardiothoracic and Vascular \\ Surgery, University Hospital of Heraklion, University of Crete Medical School, Heraklion, Greece
}

A 73-year-old man presented with bilateral chronic limbthreatening ischemia due to infrarenal aortoiliac occlusion. The ankle-brachial index was 0.2 bilaterally. Although the lesion's anatomic configuration would ideally require surgical revascularization, the patient was not eligible for bypass because of extensive skin ulceration in lower abdominal wall due to pyoderma gangrenosum (Fig. 1); therefore, endovascular recanalization was planned. First, the right brachial artery and both common femoral arteries (CFAs) were punctured. Digital subtraction angiography (DSA) revealed total occlusion of infrarenal aorta and both common iliac arteries (CIAs). Transversing left CIA occlusion through left CFA, the hydrophilic guidewire was advanced to the subintimal space. This did not lead directly to the occluded aorta; rather, it passed to the contralateral $\mathrm{CIA}$ and reentered the true lumen of the right external iliac artery (EIA). After

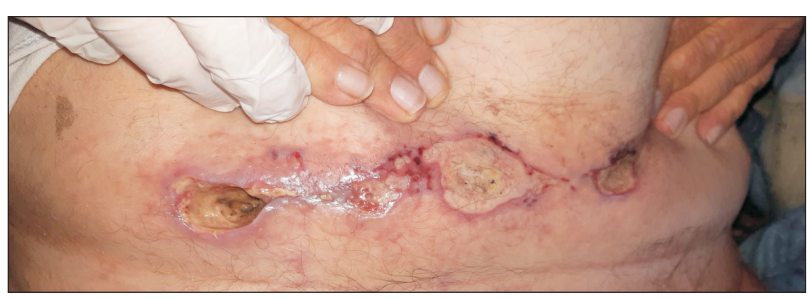

Fig. 1. Photograph showing an extensive skin ulceration in the lower anterior abdominal wall due to pyoderma gangrenosum as indicated by the pathological examination. guidewire externalization through right CFA, a catheter was advanced from right CFA and a new hydrophilic guidewire was inserted from left CFA. Subsequently, both guidewires were deployed in the same subintimal space in the occluded aorta. Using a narrow-looped configuration and supported by an angiographic Bern catheter, the guidewire was advanced into the true aortic lumen proximal to the occluded segment. Pre-dilatation was performed using two percutaneous transluminal angioplasty (PTA) balloons $(6 \mathrm{~mm} \times 100$ $\mathrm{mm})$. Subsequently, two covered stents $(6 \mathrm{~mm} \times 100 \mathrm{~mm})$ were deployed across the aortoiliac occlusion in a kissing fashion. The patient underwent additional PTA and stent deployment in stenotic lesions across EIAs. Completion DSA showed excellent flow restoration (Fig. 2). After recanalization, the patient's symptoms were resolved and ankle-brachial index increased to 0.8 bilaterally. The patient was discharged 10 days later, after improvement of the abdominal wall ulceration, which healed completely after two months. At that time, computed tomography angiography revealed excellent stent patency without signs of restenosis (Fig. 3).

Surgical revascularization is generally recommended for patients with extensive aortoiliac disease. However, endovascular revascularization may be a safe and effective treatment option for patients with contraindications for surgery $[1,2]$. A bilateral approach using the same subintimal space may facilitate reentry into true lumen.

Received July 16, 2021, Revised August 29, 2021, Accepted September 9, 2021, Published online September 28, 2021 Corresponding author: Nikolaos Galanakis, Interventional Radiology Unit, Department of Medical Imaging, University Hospital Heraklion, University of Crete Medical School, Lili Zografou 4, Heraklion 71307, Greece

Tel: 30-2810-392033, Fax:30-2810-392460,E-mail: nikos_meduoc@hotmail.com, https://orcid.org/0000-0001-9878-6053

Copyright (c) 2021 The Korean Society for Vascular Surgery

This is an Open Access article distributed under the terms of the Creative Commons Attribution Non-Commercial License (http://creativecommons.org/licenses/by-nc/4.0) which permits unrestricted non-commercial use, distribution, and reproduction in any medium, provided the original work is properly cited.

Cite this article; Vasc Specialist Int 2021. https://doi.org/10.5758/vsi.210053 

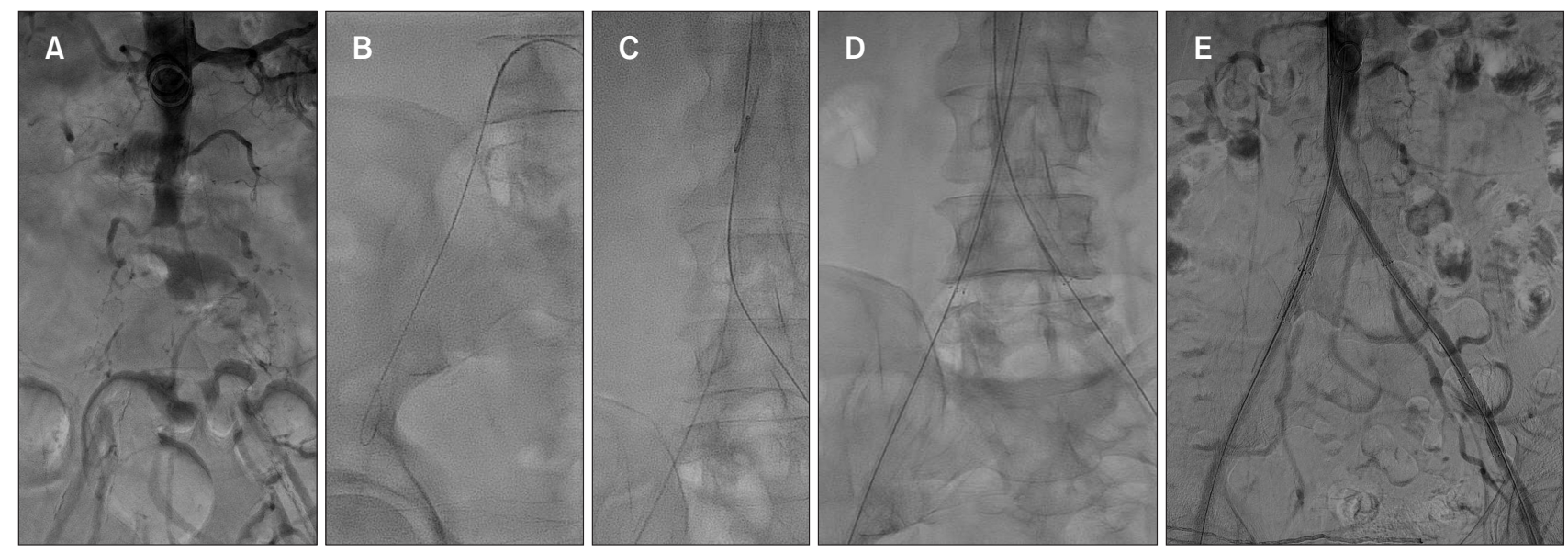

Fig. 2. (A) Digital subtraction angiography (DSA) depicted total occlusion of the infrarenal aorta and bilateral common iliac arteries and reconstruction of their distal portions via collaterals a few millimeters before their bifurcation. (B) Transversing the left iliac artery occlusion, the guidewire was advanced to the subintimal space, directed to the contralateral right common iliac artery and reentered the true lumen of the right external iliac artery. (C) After bilateral reentry attempts, the guidewires were inserted into the true lumen of the aorta just a few millimeters above the occlusion. (D) After predilatation, kissing stenting was performed across the aortoiliac occlusion using two vascular covered stents. (E) Final DSA showed excellent flow restoration.
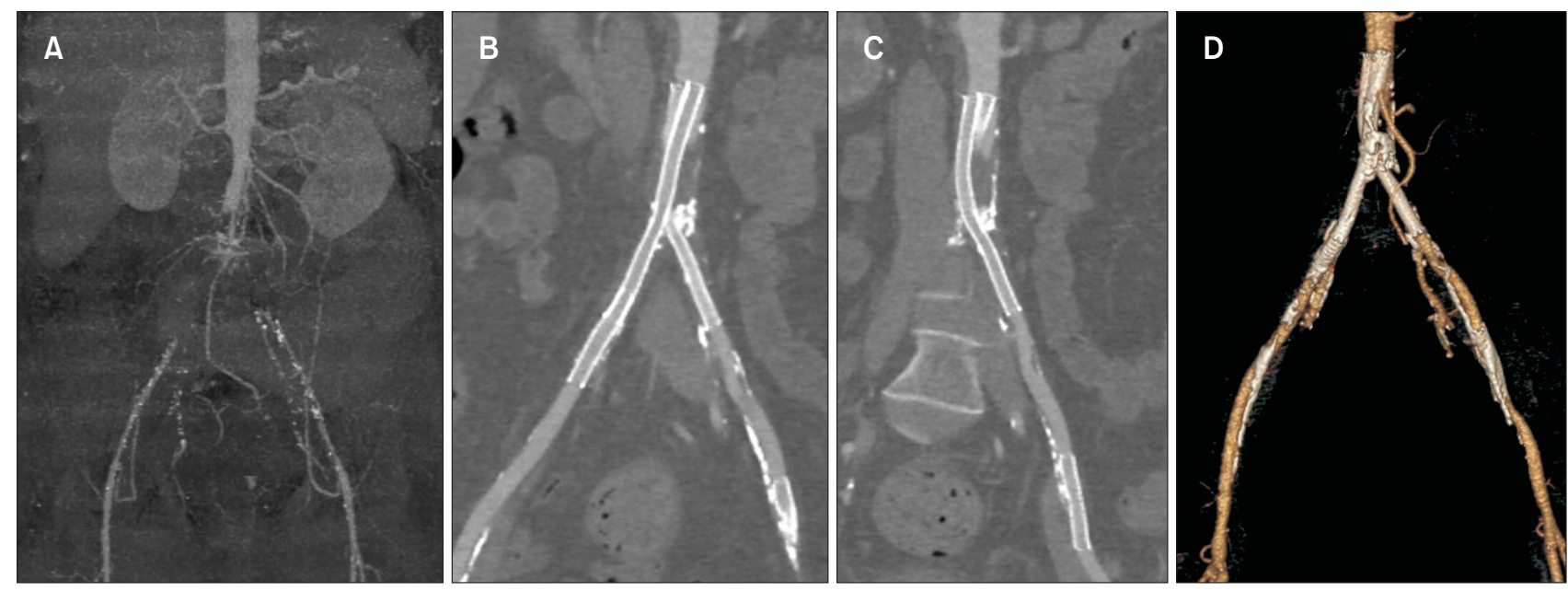

Fig. 3. (A) Computed tomography angiography (CTA), coronal maximum intensity projection (MIP) before endovascular recanalization showed total occlusion of the infrarenal aorta and common iliac arteries. Two months later, CTA curved MIP for the right (B) and left lower extremities (C) and three-dimensional reconstruction (D) revealed excellent stent patency without findings of restenosis.

\section{REFERENCES}

1) Conte MS, Bradbury AW, Kolh P, White JV, Dick F, Fitridge R, et al. Global vascular guidelines on the management of chronic limbthreatening ischemia. J Vasc Surg 2019;69(6S):3S-125S.e40.

2) Aboyans V, Ricco JB, Bartelink MEL, Björck M, Brodmann M, Cohnert T, et al. 2017 ESC guidelines on the diagnosis and treatment of peripheral arterial diseases, in collaboration with the European Society for Vascular Surgery (ESVS): document covering atherosclerotic disease of extracranial carotid and vertebral, mesenteric, renal, upper and lower extremity arteriesEndorsed by: the European Stroke Organization (ESO)The Task Force for the Diagnosis and Treatment of Peripheral Arterial Diseases of the European Society of Cardiology (ESC) and of the European Society for Vascular Surgery (ESVS). Eur Heart J 2018;39:763-816. 